\title{
The Changing Role of the Educational Video in Higher Distance Education
}

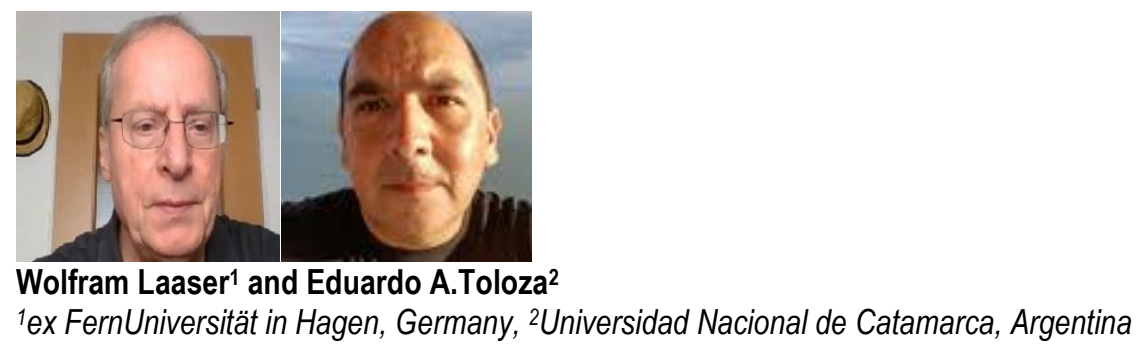

\begin{abstract}
The article argues that the ongoing usage of audio visual media is falling behind in terms of educational quality compared to prior achievements in the history of distance education. After reviewing some important steps and experiences of audio visual digital media development, we analyse predominant presentation formats on the Web. Special focus is put on recent development of new ways to generate graphics and to create animation sequences, as well as on the video presentation formats used in MOOCs. We conclude that the "new" features are in no way disruptive innovations in distance education and that the potential of video has not been sufficiently exploited. Adequate incentives to use these media for collaborative learning have not been provided and student-generated video content is at present just starting to be considered useful in instructional design.
\end{abstract}

Keywords: educational media, explainer video, distance education, video, multimedia, eLearning

\section{Point of Departure}

In the actual debate about introducing xMOOCs (i.e., Coursera, Udacity, edX and others ) as a teaching and learning environment, audio and video have been rediscovered as an important element for presenting instructional content. Following the reduction of some of the technical obstacles, and given the increasing levels of digital literacy among both students and teachers, video is now actually considered to become the dominant teaching medium on the internet. In a recent report, Kaltura (2015) presented this vision concerning the future of educational video on the Web:

- Video will be a standard part of education. Video's role will grow beyond delivering content to students, serving purposes of communication, feedback, student's assignments, and portfolios.

- Video will continue to enable flipped learning and distance learning.

- Video will enable innovative types of elearning and teaching. 
- Video will replace most textbooks.

These statements listed above are not isolated opinions but are also shared, at least in part, by a number of other writers as well (Hansch et al., 2015; Seaton, Nesterko, Mulloney, Reich, Ho, \& Chuang, 2014). In light of the history of using audio visuals in education, and given the ups and downs of their technological advances and practical uses, the following discussion considers video's comparative attractiveness and efficiency of video as a teaching medium.

\section{Audiovisual Media Development in the Past}

The large scale use of audio visual media in education started after the Second World War. However, the dominant way to deliver educational audiovisual programmes was by use of radio and later also by TV in an analogue mode. The upcoming "distance teaching universities" were forerunners in the development of the use of audio-visual material in education; they employed a variety of media to reach their remote learners. Nevertheless, the core medium in distance education was the printed text for selfstudy. The conventional universities at that time stayed with chalk and blackboard in face-to-face classroom teaching.

This panorama changed with the introduction of audio and videocassettes which better related to the printed modules. However, copying the recordings and subsequently distributing them to the students was particularly costly and constituted a logistical challenge. Therefore, they had to be classified as nonobligatory teaching aids. Acceptance of audio and videocassettes was, nevertheless, quite positive on the part of the students but not very attractive to university teachers. Firstly, most teachers were not used to being recorded. Secondly, they had no comfortable access to editing tools at school or private home. Third, they were dependent on technical staff and equipment, and lastly, they were neither given special remuneration or job promotion, nor did they receive regular training in the use of audio-visual techniques for content presentation. Therefore, the production was, to a considerable degree, influenced by the producer rather than by an academic subject matter expert.

With the digitalization of audio, and later of video, audiovisuals could be widely freed from the high distribution cost and could be integrated with and easily linked to other digital media. For the first time, integration of text, still images, sound, and video on a single data carrier (CDR and later DVD) became feasable. Creative projects of high didactic and technical quality showed the way to meaningful and exciting media production. (For a description of educational programs on DVDs, see Laaser \& Sieg, 2004.) However, specialized staff and a complete video or TV studio had to be available, as well as the appropriate financial means. Therefore, it is not surprising that relatively sophisticated multimedia productions needed a long project duration, sometimes even taking two to three years. Due to limited usage by students, because CD or DVD productions were usually an add-on and not obligatory teaching material, and due to a lack of promotion by the university administrations, multimedia products had only a relatively small impact on students' learning. The good thing about these multimedia projects was the intensive trial and error process of finding out attractive and suitable design patterns and visualization concepts.

However, after the intensive public and private funding of multimedia projects, the expectation of saving costs by replacing traditional lecturing with massively distributed and shared content, which the educational ministries had clung to, did not materialize. In addition, the network capacity and speed of the Internet was not yet sufficient, so that the focus shifted away from sophisticated, but isolated 
multimedia, to integrated text-based digital courses on the Web. Furthermore, for economic reasons, videos became shorter and were recorded with small and flexible camera teams instead of using big recording studios equipped with physical studio decoration. The decrease in public and private funding also led to reductions of external shootings off-campus in favour of recording lecturers with a fixed camera and greenscreen background images or by producing interviews just by changing the camera position while recording the interviewer and the intervieww separately. The quality of educational video declined. A number of multimedia institutes were shut down at that time. The typeicla Web video became shorter, simple, and integrated with other Web content.

As to the multimedia productions mentioned, they did not permit any sort of simultaneous communication. Therefore, a parallel development strand is also important as well: the one from textbased chat software to multipoint videoconferencing systems. The integration of digital text, audio, and video reduced the need to produce stand-alone products and directed the focus on the related course context including comments and discussions.

Some shortfalls of past media development, such as high production and distribution costs, and the integration of digital text, audio, and video have been overcome. Although it must be mentioned that the teachers' and students' lack of knowledge on how to conceptualize and produce good quality videos, by themselves, led most academics to rely on professional support and equipment or refrain from using the medium. For these reasons, user-created content remained scarce.

\section{Actual Use of Web Videos}

\section{Explainer Videos as an Additional Option}

The so called explainer videos (Powtoon, 2016; Philippi, 2016) are an upcoming genre that may have some similarities with the Asian anime cartoon-type of storytelling. Animations have always been very popular with young kids and have a long tradition that goes back to Donald Duck cartoons. However, this type of animation videos has not been applied to formal or informal education because of the development cost involved in designing each frame seperately.

The presentation of digital videos in the framework of Web pages has fueled the development of new ways of content presentation and also the incorporation of educational content. Today, a typical application on open Websites, such as YouTube, is about how to do something, for example, how to play the guitar, how to prepare a meal, or how to go about matriculating. Big companies are starting now to use videos for staff training instead of courses taken from learning management systems.

Short animation videos applied to education are at present labeled explainer videos, which is not really a very elegant choice because most educational videos try to explain something.

We can very broadly list three types of animation videos:

Stop motion. The first type follows a pattern that stems from the early production of animation films. The camera takes single shots of drawings or objects. The object or drawing changes with every shot (frame) and, when played in a short time interval, creates the sensation of motion. To produce a clip in its most simple format we can use for example a handwritten text or drawing on a tablet, or by moving Play People figures or objects formed by plasticine. In addition, a camera and a 
device to record an underlying narration are needed. Therefore, a smartphone with a basic editing software such as Apple's iMovie is the minimal equipment required.

Use of ready made templates. A second type of simple animations uses ready made templates for a great number of objects that can be animated by changing a set of different attributes, for example, a human character with hands up or down, handshake, laughing, or a sad look, and so on. The producer's contribution is to select the single images or preprogrammed movements from the menu and place them on a timeline which usually incorporates also an audio track for the spoken commentaries. Simple scriptwriting prior to the actual recording is a useful preparation. Specific programming knowledge though is not needed.

Programming with Flash or HTML5. As a third form of animated video, we will mention two dimensional animations programmed with software like Flash or HTML5. In Flash, for example, the movement of objects is provided by setting the first and the final keyframe. The software then calculates the frames needed to show the transition.

When employing three dimensional virtual environments, advanced programming languages, such as Java 3D, are needed to model virtual worlds (Secondlife, Wonderland).

We will concentrate on the first animation types for the simple reason that they are quite possibly easier for teachers and learners to handle than programmes that require programming knowledge would be. The list of programs that are shown below represents only a fraction of the existing variety of simple Web tools for creating explainer videos:

- Powtoon

- Moovly

- Go Animate

- Videoscribe

- Snagit

- Explain Everything

- AdobeVoice (iPad)

- PuppetPals (iPad)

- Papershow for Teachers (alternative instead of iPads)

- Sketchbook pro (tool for drawing if tablet is not available)

Powtoon (2016) gives the following tips for creating clips with their software:

- Keep your message short and positive. 
- Be sure your message sticks by using techniques like rhyme, repetition, catchy music, and relatable characters.

- Have the confidence to leverage social proof that demonstrates how your brand or your product helps your tribe.

If we look at the present applications, we will find that most of them are just a visual illustration of the audio commentary - an illustration that has been created by animated images. If the images were to be played without listening to the parallel audio as well, the pictures chosen from templates would not help much in giving new insight into a problem and might even be completely incomprehensible. The importance is laid mainly on getting the attention of the student rather than explaining something by means of the visual representation. There is a constant, mostly quite rapid, presentation speed and a permanent, but not varying, presentation format. It is therefore easy to understand that the length of the clips is usually very short, which, at the same time, limits the possibility of explaining complex and perhaps conflicting issues. For more sophisticated animated videos, a specialized designer would have to support the content expert.

There are some positive aspects of the so-called explainer videos which are probably better described as video presentation of animated text or image rather than be labelled as explainer video. First, they can form part of a larger educational video as a motivating, if short, interruption to the presentation mode. Another aspect is that the introduction of simple techniques, such as creating a podcast or a short handmade animation video, can be a good starting point in training teachers or students to create meaningful videos, e.g., how to enroll or how to use the university library. However, the fun effect of the animation techniques can also provoke the illusion that learning is extremely easy and that the content presented is a fast-track to acquiring knowledge.

\section{Video Presentation Formats in Blended Learning and MOOCs}

One of the design patterns most often used at universities at present is the lecture capture format, where a teacher's lecture is recorded by a fixed camera, showing either his "talking head" or his writing on a tablet or whiteboard; sometimes shots of the audience are added. In contrast to the extremely short explainer videos a recorded lecture runs about 45 minutes. Thus, this particular format is not in line with some recommendations for producing short educational videos. A recorded lecture may, however, be split into smaller chunks which will require thoughtful planning of the lecture and some basic editing. 


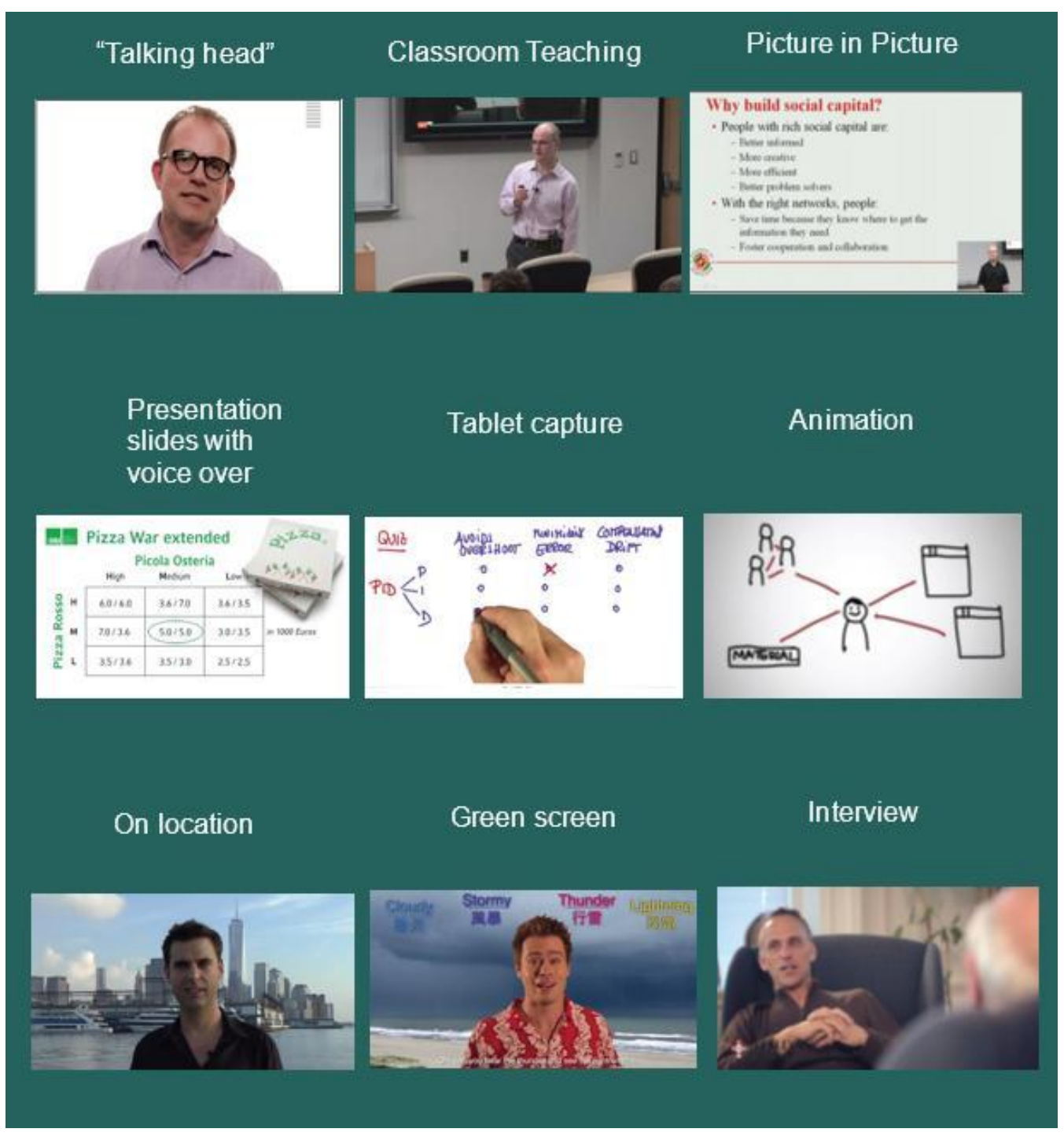

Figure 1. Video design patterns. Source: modified from Hansch et al. (2015).

The so-called lecture capture format is increasingly used and praised as a tool, which provides students access to a recorded lecture at any time and helps teachers to concentrate on interactive teaching during their tutorial face-to-face activities. The model in which lectures are recorded whereas tutorials are run in face-to-face format, is called the flipped classroom. The media concept behind the flipped classroom does not really represent an educational innovation which improves understanding of new and nontrivial content, What it really does is to replace the taking of lecture notes and reading assignments with a pre-recorded or streamed lecture. Basically it helps to cement an already-outmoded teaching model (Baggaley, 2015). Ian Bogost (2013, para. 10) expressed it like this:

Perhaps surprisingly, a flipped classroom doesn't fundamentally alter the nature of the experience in the way that McLuhan and Davidson propose. Both MOOCs and flipped classrooms still rely on the lecture as their principal building block. In a typical classroom students listen to lectures. In a flipped classroom, students still listen to lectures - they just do so as homework, edited down into pleasurably digestible chunks. The lecture is alive and well, it's just been turned into a sitcom. 
It is also quite obvious that this presentation format covers only a very small part of the possible educational applications of video. It has, moreover, the disadvantage that the teacher may feel uncomfortable about being recorded and anxious not to make mistakes and that way delivers teaching which is less authentic than his classroom lectures normally would be. Sometimes the lecture text is just read from a teleprompter.

In general, video can be used for a number of purposes other than just simple lecture capture activities. An interesting classification is given by Schwartz and Hartmann (2007). These authors distinguish between the different uses of video according to "seeing, doing, engaging and saying" (p.7). "Seeing" refers to the things we know. but cannot observe directly; doing refers to skills, such as training in how to use laboratory equipment, perhaps using detailed shots or slow motion; engaging is used to motivate a person by creating emotional reactions; and saying is stressing the expository character of video applications. Similar classifications can be found in Bates (2015) and Koumi (2006).

The use of video in education was pushed by the massive open online courses produced by famous American universities - but the presentation format, especially of the xMOOCs, was mainly of the directly lecture capturing type. The main reason for the relatively poor design in actual MOOCs is certainly due to the institutions" objective which were to keep costs at a minimum and to guarantee fast production, especially as MOOCs initially have been offered free-of-charge. This is true at least in the case of XMOOCs (Daniel, 2012). The cost of their video productions still represents the key cost item in MOOCs, although the format is kept very simple. It seems that the design of massively open online courses and lecture capture somehow brings educational video back to the level of the Chinese talking head approach, which was used to train millions of teachers in China by radio and television. For example, 1.7 million students were enrolled at the China Central Radio and TV University between 1979 and 1986 (Laaser, 1999).

In that way, the style of educational videos on the Web is rather "fast and simple"rather than showing thoughtful and elaborated productions. The cartoon type of explainer videos is limited to easily understandable short topics and is more entertainment than a serious academic presentation. The educational Web video, in general, often lacks detailed descriptions about the background and context of the respective production.

Another influence on designing educational videos comes from multimedia platforms, such as YouTube, which are open for everybody to upload self-created video material onto the Web. Consequently, the quality standards are lowered and adjusted to the technical possibilities of the producer/consumer, who usually only has a smartphone, a Web cam, or a camcorder at their disposal to record and edit videos using available Web tools at no cost.

It is therefore not surprising that many insights and experiences gained from past educational filming are neglected or unknown. For example, the principles of the Theory of Perception say that learning only happens after perception or that a permanent stimulus loses its effect if there is no variation. Many educational videos are extremely fast in presenting their message and use techniques like speeding up handwritten drawings or handmade paper works to catch the attention of the viewer. Often the content is being presented as easy to learn and does not point to alternative views or offer links to related bibliography, unless additional sales of related products are being promoted.

As perception is selective, the aspect, which is most relevant in a scene or picture (teacher, text, or image) has to be focussed. Rigid models that maintain a fixed layout, for example. Picture- in-picture, do not 
fulfill this requirement. For fear of losing the attention of the learner, the duration is usually short. If the video clips which the student has selected are from different sources, their design patterns will change from one video to the next. This also means that the scattered content is not easily appropriated by the student and burdens him/her with the additional workload of perceiving and decoding the content and then relating the video to his/her individual learning context.

With regard to graphics design, some basic rules of Gestalt psychology are not taken sufficiently into consideration. For example, that regions and meanings have to be clearly distinguishable, or that simplicity, symmetry, and regularity are considered to be good forms (Laaser, 1984).

This is valid also for the recent development of Infographics (Karbach, 2012; Vest, 2009). Infographics are automatically created visual representations of data, for example, time series or data about remuneration in different occupations and so force. The representation of word counts in Wordle is a well known example of infographics. Like in the case of explainer videos, these Webtools are based on templates which include some options with which to modify the graphics design but these options are quite limited and may, if they have been combined from different sources, contribute to a scattered and heterogeneous design of the educational video.

Andrew Keen criticises the lack of expertise in his book The Cult of the Amateur (2007). On the other hand, the preconditions to produce interesting and creative user-generated content are given due to easier to use low-cost recording and editing systems. However, students and teachers alike still need quite a considerable amount of training and motivation to make them get involved with time-consuming video production. Podcasts would clearly be a perfect model to start training on how to produce educational audiovisuals. Similarly, the production of simple explainer videos can be learned with little prior knowledge. Furthermore, the concept of awarding a single learner`s achievements could be extended to badges for teachers, which, in turn, would help them to qualify for job promotion.

\section{The Efficiency and the Value Added of Web-Based Video}

Historically, the research of value-added created by videos has been not very frequent. The main question was as to whether video is in any way more or less effective as a method of teaching than other media. We usually hear statements such as "a good image is worth a thousand words" or that "seeing is less effective than doing." However, it is extremely difficult to prove any such statements. This is due to the dialectical relationship between form and content. Form cannot exist without content and content not without form. Most studies that compared, for instance, print with radio, video, and television, leaving the content unchanged, did not reach a clear conclusion (Laaser, 1986).

As Dwyer had already pointed out in 1967, each medium should be evaluated in terms of the learning objectives for which it is best designed. Comparison of different media in relation to one type of learning objectives, or where specific learning objectives are not identified and reported, produce results which are uninterpretable (p. 251). Similar difficulties with regard to research about effects of sound and its relation to on-screen images are reported Koumi (2003).

It is important to recognize that there are different benefits or value-added according to which stakeholder we observe: the students, the teachers, or the institution. Obviously, the students are in the position of winners-unless fees are raised for including video lectures-as they can call up parts of the digital lectures, and they can easily watch and listen to the lecture at any time they wish to, no matter 
what device (mobile, notebook, or PC) they have at their disposal. They need no physical storage of the teaching material.

Teachers may be split between those who are intrinsically motivated to try out and discover the potential and pedagogical usefulness of new media and those who are complaining about the additional workload, usually with no advantages, financial or otherwise. Finally the institution will compare the cost of the media application and the cost of training the staff with benefits such as additional prestige or higher enrolment figures, though this economic reasoning is not unique to the medium video.

However, a 50\% dropout in watching MIT's XMOOC videos is an alarming sign that the use of video may not match students' expectations (Seaton et al. 2014). A recent study about how MOOC videos affects students engagement comes to the following conclusions (Guo, Kim, \& Ruby, 2014, p. 2):

- Shorter videos are more engaging than longer videos.

- Videos that intersperse an instructor's talking head with slides are better than using slides alone.

- Videos with a personal touch can be more engaging than high-quality studio recordings.

- Khan (Academy) style tablet drawing tutorials are more engaging than PowerPoint slides or code screencasts.

- Videos, which show instructors speaking quite quickly and with enthusiasm, are more engaging.

The research methodology of Guo et al. (2004) is grounded on the length of time students spend watching a video and on the frequency of trying to solve a multiple choice test within 30 minutes after viewing. Although the methodology of the research is quite sophisticated, most of the results correspond to common sense and are not very surprising.

Another approach is the use of eye tracking methodologies to analyse how students direct their attention towards certain screen elements (hotspots) (Scharma et al., 2014). However, the interpretation of the data and their ability to draw respective didactic conclusions are still open issues.

\section{Some Conclusions and Perspectives About the Future of Educational Video}

What can be learned from the discussion? First of all, the current use of video is actually deficient in terms of exploiting its media characteristics. Concepts such as XMOOCs and flipped classroom in this respect are not a step forward, but a step backwards in the deveopment of educational video. One medium is replaced by another without major changes in the content. Furthermore, especially high enrolment numbers, such as in MOOCs, need special support. If high enrolment goes with non-existent teaching staff or too few tutors to provide the necessary individual support, students are left on their own and are inclined to drop out. This support can only be replaced up to a certain degree by peer review and mutual cooperation. Students will have to invest too much of their time before they are able to identify information that is credible and relevant to them. In particular, students coming from different 
cultures and learning experiences will not all react to a given presentation format in the same way (Jadin \& Gaisch, 2014), which makes support even more decisive.

Educational video can play a role in documenting the individual environment and students' contributions. These individual student-produced videos could be uploaded onto a server, and in that way, ease the search for cooperation and mutual understanding amongst the extremely heterogeneous clientele of MOOCs. However, any feedback mechanism will increase cost for institutions that offer participants such options.

It is also important to research the media use that students make in their private environment and to link that to the style and format of educational video presentations. Students and teachers should learn to produce videos for themselves, and to gain an idea about what is useful and feasible for them. According to the Kaltura Report, the active use of video by students is still in its infancy (Kaltura, 2015). Videos can document the reality, imagination, and feelings around them and to relate them to their individual experience.

It is certainly helpful, but not sufficient, to include some interactive exercises into a "lecture capture" video thus making it more interactive or improvinge search algorithms to trace segments of the content of a video directly (Woll, Buschbeck, Steffens, Berrang, \& Löviscach, 2014).

The need to relate the theoretical context to real world experience is neglected, although video can contribute a lot to connect both views. Producing videos on your own implies that you have to come into direct contact with objects, people, situations, and processes, not by just watching another video or reading a book. It is important to leave the halls of the university and to connect to your environment (Baumann \& Jahn, 2015). Projects in which students use smartphones to record, to classify, and to report or act are pointing to the needed change of the media use (Kukulska-Hulme \& Traxler, 2005; Islas Sedano, 2012).

The future of video on the Web will depend on the stakeholders' reactions: to what extent will students, teachers, and institutions appropriate and discover the medium for themselves? The professor dominates-at least in the lecture capture model-a huge part of the teaching and learning process, and may therefore prefer his/her traditional role instead of playing the role of a facilitator. The reasons for some resistance are already known from the past. New career schemes and interesting training opportunities will be necessary to change their preferences. Competitive elements such as presentation of the best use cases can also be helpful. Distance universities, just as other big institutions, will aim, however, according to their own objectives, at global marketing to raise enrolment and economic return.

On the global scene, we will therefore experience an increasingly fierce competition among providers of Web-based offers based on standardized production schemes including educational video, which may lead to the rising influence of multinational providers of audiovisual educational material. The role of the teacher in the use and production of educational video will be split among specialized providers of content, design, production, presentation, and marketing. User-created content will not be the main focus on the agenda.

This implies that small players will have to concentrate on the specifics of their cultural environment. The educational video will remain certainly an important medium for transmitting educational content, but it will be merged even closer with other media such as print, animation, geo-location and sensor-led technologies, simulation, games, and conferencing, thus the differences between single media will 
vanish and we might enter into a period of "industrialisation"and "standardized production and design" of educational video.

\section{References}

Baggaley, J. (2015). Flips and flops. Distance Education, 36, 437-447. doi: 10.1080/01587919.2015.1041677.

Bates, A. W. (2015). Teaching in the Digital Age [Online book]. Tony Bates Associates Ltd. Retrieved from https://opentextbc.ca/teachinginadigitalage/

Baumann, C., \& Jahn, D. (2015). Film in der Hochschullehre [Film in Higher Education] Hochschuldidaktische Aufsätze 2/2015. FBZHL, University of Erlangen

Bogost , I. (2013). The condensed classroom [Blog Post]. The Atlantic. Retrieved from http://www.theatlantic.com/technology/archive/2013/08/the-condensed-classroom/279013/

Daniel, J. (2012). Making sense of MOOCs: Musings in a maze of myth, paradox and possibility. Journal of Interactive Media in Education, 2012(3). Retrieved from http://jime.open.ac.uk/articles/10.5334/2012-18/

Dwyer, F. M. (1967). Adapting visual illustrations for effective learning. Harvard Educational Review, $37,1967$.

Guo, P. J. Kim, J., \& Rubin, R. (2014). How video affects student engagement: An empirical study of MOOC videos. L@S 2014 March 4-5 2014, Atlanta, Georgia

http://dx.doi.org/10.1145/2556325.256639

Hansch, A., Hillers, L., McConachie, K., Newman, Ch., Schildhauer, T. \& Schmidt, P. (2015). Video and online learning: Critical reflections from the field. Alexander von Humboldt Institute for Internet \& Society. Discussion Paper Series, 13. March 2015 Retrieved from http://papers.ssrn.com/sol3/papers.cfm?abstract id=2577882

Islas Sedano, C. A. (2012). Hypercontextualized Games, Dissertations in Forestry and Natural Sciences No. 79, University of Eastern Finland, Joensuu

Jadin, T., \& Gaisch, M. (2014). Extending the MOOCversity. A Multi-layered and diversified lense for Mooc Research. Proceedings of the European MOOC Stakeholder Conference Summit 2014, U. Cress (Ed.), 73-79 Retrieved from https://www.emoocs2014.eu/sites/default/files/Proceedings-Moocs-Summit-2014.pdf

Kaltura (2015). The state of video in education. New York: Kaltura. Retrieved from http://site.kaltura.com/rs/984-SDM859/images/The State of Video in Education 2015 a Kaltura Report.pdf 
Karbach, M. (2012). Eight tools to make awesome graphics: Educational technology and mobile learning [Blog Post]. Retrieved from http://www.educatorstechnology.com/2012/05/eightfree-tools-for-teachers-to-make.html

Keen, A. (2007). The cult of the amateur. New York: Doubleday.

Koumi, J. (2003) Synergy between audio commentary and visuals in multimedia packages. Journal of Educational Media, 28(1).

Koumi, J. (2006). Designing video and multimedia for open and flexible learning. London, New York:Routledge

Kukulska-Hulme, A. \& Traxler J. (2005). Mobile Learning. London, New York:Routledge

Laaser, W. (1984). Didactic design of video instruction in distance education. Epistolo didaktika 1984/1\&2, 4-15.

Laaser, W. (1986). Wirkung alternativer Gestaltung von Studienmaterialien bei formalen ökonomischen Problemstellungen .[Effects of alternative design of study material in economics], Zeitschrift für erziehungswissenschaftliche Forschung, 1986(1).

Laaser, W. (1999). Technologies for distance education in developing countries. In S. Mitter, M-I Bastos, \& A. Bartzokas, (Eds.), Europe and developing countries in the world information economy. London, New York: Routledge.

Laaser, W., \& Sieg, I. (2004, January). DVD - A new component in educational technology. Learning Technology Newsletter, 6.

Philippi, B.S. (2016). Erklärvideos selbst erstellen. [Creating explain videos by yourself] ETeaching.org., Video Special 2016. (recorded lecture) Retrieved from https://www.eteaching.org

Powtoon. (2016). How to make an animated explainer video [Blog Post]. Retrieved from https://www.powtoon.com/videomarketing/animated-explainer-video/

Schwartz, D. L. \& Hartmann, K. (2007). It is not television anymore: Designing video for learning and assessment. Stanford University. Retrieved from http://aaalab.stanford.edu/papers/Designed_Video_for_Learning.pdf

Seaton, D. T., Nesterko, S., Mullney, T., Reich, J. Ho A., \& Chuang I. (2014). Characterizing video use in the catalogue of MITXMOOCs. Proceedings of the European MOOC Stakeholder Conference Summit 2014, U. Cress (Ed.), 140-146

Vest, J. (2009, December 2). Six steps to creating high quality video training [Blog Post]. Learning Solutions Magazine. Retrieved from http://www.learning solutionsmag.com/articles/185/

Woll, R., Buschbeck, S., Steffens, T., Berrang, P., \& Löviscach, J. (2014). A platform that integrates quizzes into video. Proceedings of the European MOOC Stakeholder Conference Summit 2014, U. Cress (Ed.), 155-159. 
The Changing Role of the Educational Video in Higher Distance Education Laaser and Toloza

Athabasca

University

(c) (7) 